\title{
Trends, microbiology, and outcomes of infective endocarditis in children during 2000-2010 in the United States
}

\author{
Shipra Gupta, MD1 | Ankit Sakhuja, MD2 ～Eric McGrath, MD ${ }^{1} \quad$ Basim Asmar, MD1
}

${ }^{1}$ Department of Pediatrics, Wayne State University School of Medicine, Division of Infectious Diseases, Children's Hospital of Michigan, Detroit Michigan, USA

${ }^{2}$ Division of Nephrology, Department of Internal Medicine, University of Michigan, Ann Arbor, Michigan, USA

\section{Correspondence}

Shipra Gupta, MD, Division of Infectious Diseases, Department of Pediatrics, Children's Hospital of Michigan, Detroit, Michigan 48201.

Email: drshipragupta@yahoo.co.in

\begin{abstract}
Background: We studied the incidence, trend, underlying conditions, microbiology, and outcomes of infective endocarditis (IE) in children during 11 years using Nationwide Inpatient Sample (NIS) database. This is the largest all-payer inpatient care database in the United States containing data for more than 8 million hospital stays from over 1000 hospitals.

Methods: NIS data from 2000 to 2010 of primary discharge diagnosis of IE in children aged $\leq 19$ years old were studied. Children with underlying congenital heart defects and acquired heart conditions were identified. Microbiological causative agents were recorded. Linear regression was used to assess trend of incidence over time.

Results: An estimated 3,840 (95\% Cl: 3,395-4,285) children had a discharge diagnosis of IE. The overall incidence was 0.43 per 100000 children. The incidence was stable over the study period ( $P=.4$ for trend). The majority of patients $56.2 \%$ were $\geq 11$ years old and $15.4 \%$ were $\leq 1$ year. Underlying cardiac conditions were present in $53.5 \%$ of patients. Overall $30.2 \%$ of cases were culture-negative. Among those with identified pathogens, Staphylococcus species were most common (43.1\%) followed by Streptococcus species (39.5\%). Viridans Streptococcus group was most common in those with underlying heart disease (32.7\%) and S. aureus was most common in those without heart disease (46.9\%). Among culture-positive patients, there was a decline in proportion of Staphylococcal IE $(P=.03)$ and an increase in proportion of Streptococcal IE $(P=.04)$. Overall mortality was $2.8 \%$. Patients with Staphylococcal IE had longer median length of stay (12 vs. 9 days; $P<.01)$ and the highest mortality $(4.7 \%)$.

Conclusion: The incidence of IE in children has remained unchanged in the United States during the 11-year study period. Among culture-positive patients there was a significant decrease in Staphylococcal IE and a significant increase of Streptococcal IE. Staphylococcal IE was associated with increased LOS and highest mortality.
\end{abstract}

\section{KEYWORDS}

infective endocarditis, pediatrics, epidemiology, microbiology, outcomes

\section{1 | INTRODUCTION}

During the past decade there has been an increase in the risk factors for infective endocarditis (IE). The advancements in cardiovascular surgery have led to improved survival of children with congenital heart disease (CHD) beyond infancy and into adulthood. ${ }^{1}$ These children are at increased risk of developing postoperative infections including IE due to presence of prosthetic devices placed for correction or palliation of heart defects. ${ }^{2-5}$ There has also been an increase in the use of central venous catheters in children for medication infusion and blood drawing, thus increasing the risk of catheter-related infections, including IE. ${ }^{6}$ With the increase in these risk factors, there is an expected increase in incidence of endocarditis. Also, the advancements in microbiologic techniques has lead to improved recovery and better identification of organisms in blood and tissue cultures, thereby improving the etiologic diagnosis of previously culture-negative labeled IE cases.

The overall incidence of endocarditis has increased during the past several years. In a recent report by Bor et al., during the period 1998- 
2009 hospital admissions for IE in the United States rose from 25511 (9.3 per 100000 population) to 38976 (12.7 per 100000 population). The endocarditis admission rate increased $2.4 \%$ annually after adjustment for population growth and aging. Pediatric cases ( $<18$ years) accounted for $1.9 \%$ of total cases. ${ }^{7}$ In a more recent report by Pant et al., based on national data for the period 2000-2011 the incidence of IE increased from 11 per 100000 in US population to 15 per 100000.8

In children, hospitalization rates due to infective endocarditis (IE) are lower as compared to adults. One report from a single institution estimated that IE cases during 1972 to 1982 accounted for 1 in every 1280 (0.78 per 1000) pediatric admissions annually. ${ }^{9}$ More recent data from a multicenter study estimated that there were between 0.05 to $0.12 \mathrm{IE}$ cases per 1000 hospital admissions during 2003 to $2010 .^{10,11}$ IE incidence is higher in children with underlying congenital heart disease (CHD) and was estimated as a cumulative incidence of 6.1 per 1000 children. ${ }^{12}$ There is paucity of data regarding recent national trends of IE in children. We used a nationally representative sample of hospitalized children to assess the recent trends in incidence, microbiology and outcome of infective endocarditis during the period 2000-2010.

\section{2 | METHODS}

\section{1 | Study design and data source}

We performed a retrospective study using national data from the Healthcare Cost and Utilization Project-Nationwide Inpatient Sample (NIS). NIS is the largest all-payer inpatient care database publicly available in the United States that contains data from a $20 \%$ stratified sample of U.S. community hospital. ${ }^{13}$ Each hospitalization is treated as an individual database entry and information regarding common demographic variables-age, race and sex along with primary insurance, hospital characteristics-teaching status, location (rural vs. urban), size of hospital and hospital region are available. Data from 2000 to 2010 period were used for this study. We used the provided principal diagnosis, secondary diagnoses and procedural diagnoses associated with each hospitalization in the database for this study.

\section{2 | Study population}

We included hospitalizations of patients with age $\leq 19$ years. In accordance with previous literature ${ }^{7}$ we defined infective endocarditis as designated by the International Classification of Diseases, Ninth Revision, Clinical Modification (ICD-9-CM) codes for acute and subacute endocarditis (421.X), gonococcal endocarditis (098.84), meningococcal endocarditis (036.42), candidal endocarditis (112.81) and histoplasma endocarditis $(115.04,115.14,115.94){ }^{7}$ We excluded those with noninfectious or chronic endocarditis.

\section{3 | Study variables}

We identified demographic characteristics (age, sex, and race), hospital characteristics (teaching status, location, bed size and region)

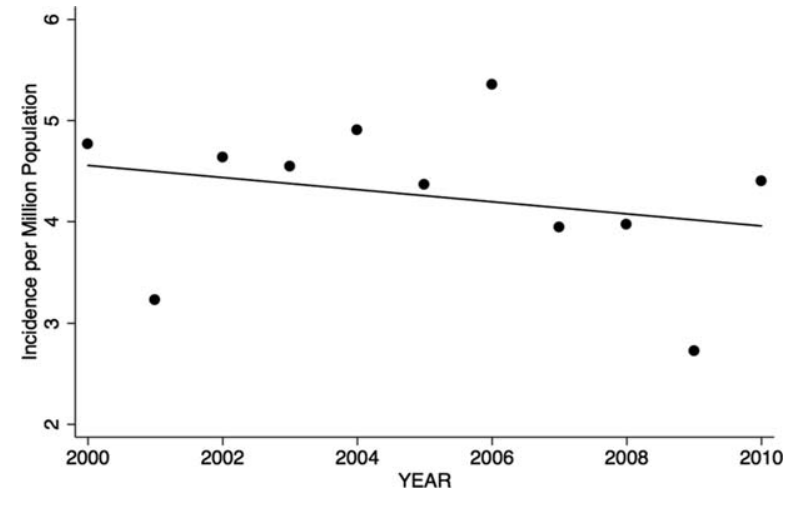

FIGURE 1 Incidence trends of infective endocarditis in US children during 2000-2010

and primary payer using appropriate variables from the NIS database. The causative organisms and predisposing cardiac conditions using ICD-9-CM codes are detailed in Supporting Information Tables S1 and S2.

\subsection{1 | Outcomes}

Our primary outcome of interest was all cause in-hospital mortality. We also looked at length of stay (LOS) for those hospitalized with IE. In addition, we looked at complications of IE during that hospitalizationacute kidney injury (AKI), stroke, mechanical ventilation use, acute myocardial infarction, central nervous system (CNS) abscess, meningitis, encephalopathy, convulsions and extracorporeal membrane oxygenation (ECMO) use. We assessed the proportion of those undergoing cardiac valve replacement during that admission. The ICD9-CM codes used to identify these complications are provided in Supporting Information Table S3.

\section{4 | Statistical analysis}

We performed all statistical analysis using STATA 13.1 (College Station, Texas, USA). Using the weights provided in NIS database, we generated national estimates for the number of overall IE hospitalizations. The incidence of IE hospitalizations per 100000 population was calculated by utilizing annual population estimates from the United States Census Bureau. We used the chi-square test to compare categorical variables and linear regression to assess the significance of trends over time. A $P$ value of $\leq .05$ was deemed significant.

\section{3 | RESULTS}

\section{1 | Incidence of IE}

An estimated 3,840 (95\% Cl: 3,395-4,285) children had a discharge diagnosis of IE. Children accounted for $2 \%$ of the total hospitalizations for IE during the study period. The overall incidence of IE was 0.43 per 100000 children and was stable over the study period $(P=.4$ for trend) (Figure 1). 
TABLE 1 Demographics of US children with infective endocarditis and treating hospital characteristics (2000-2010)

\begin{tabular}{|c|c|}
\hline Characteristic & Percent \\
\hline \multicolumn{2}{|l|}{ Age } \\
\hline $1 \mathrm{y}$ & 15.4 \\
\hline $2-5 y$ & 11.7 \\
\hline $6-10 y$ & 16.6 \\
\hline $11-19$ y & 56.2 \\
\hline Male & 54 \\
\hline \multicolumn{2}{|l|}{ Ethnicity ${ }^{a}$} \\
\hline White & 45.9 \\
\hline Black & 13.7 \\
\hline Hispanic & 13.7 \\
\hline Asian & 2.6 \\
\hline Native American & 0.8 \\
\hline Others & 4.2 \\
\hline \multicolumn{2}{|l|}{ Insurance } \\
\hline Medicaid & 37.6 \\
\hline Private & 51.5 \\
\hline Medicare & 0.7 \\
\hline Others & 4.4 \\
\hline Self-pay & 4.7 \\
\hline Teaching hospital & 81.9 \\
\hline \multicolumn{2}{|l|}{ Region } \\
\hline South & 38.7 \\
\hline West & 21.3 \\
\hline Midwest & 20.1 \\
\hline Northeast & 19.8 \\
\hline
\end{tabular}

\subsection{Study population demographics and hospital status}

The general characteristics of the study population and treating hospital are summarized in Table 1 . The majority of patients, $56.2 \%$, were $11-19$ years old and $15.4 \%$ were $\leq 1$ year old. Males comprised $54.0 \%$ of patients, $45.9 \%$ were white, $13.7 \%$ black, and $13.7 \%$ Hispanic.

Underlying cardiac conditions were present in $53.5 \%$ of patients. These subjects were mostly $\leq 10$ years old, male, and white. They were also more often admitted to teaching hospitals (84.9\%) when compared to patients without underlying cardiac conditions (78.5\%) $(P=.06)$. Among those with cardiac conditions, $81.4 \%$ had congenital heart disease, $15.2 \%$ rheumatic heart disease, $7.5 \%$ prosthetic valve, $3.9 \%$ cardiac device, and $3.6 \%$ had cardiomyopathy. The most common

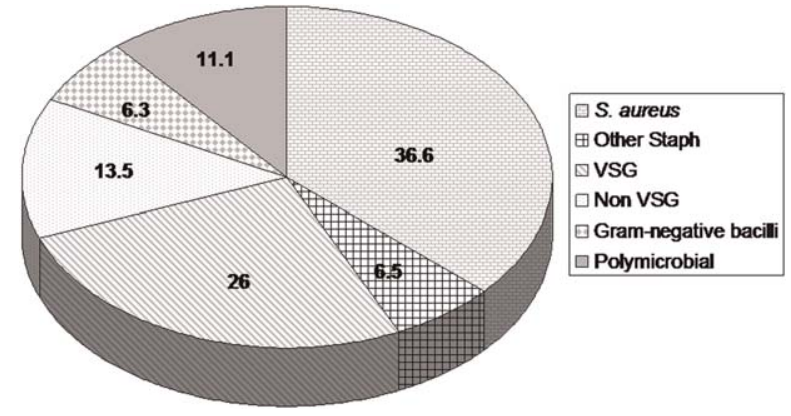

FIGURE 2 Distribution (\%) of microorganisms isolated in culturepositive infective endocarditis cases in US children during 20002010

congenital heart defect was ventricular septal defect (32.2\%). Cyanotic heart defect accounted for $25 \%$ of congenital heart defects.

\section{3 | Microbiology}

A code for any organism was not included in $30.2 \%$ of IE discharges and thus were considered culture-negative. In those with culturepositive IE, Staphylococcus species were the most common $(43.1 \%$ hospitalizations) pathogen followed by Streptococcus species (39.5\%). When categorized further, Staphylococcus aureus accounted for $36.6 \%$ of hospitalizations and viridans Streptococcus Group (VSG) for $26 \%$ whereas $11.1 \%$ of hospitalizations were coded for more than one microorganism (Figure 2). VSG was the most common pathogen in those with underlying heart condition (32.7\%) and S. aureus was most common in those without underlying heart condition (46.9\%) (Table 2).

Among patients with positive cultures, the proportion of Staphylococcal IE decreased from $46.9 \%$ in 2000 to $33.1 \%$ in 2010 ( $P=.03$ for trend) whereas the proportion of Strep spp. IE increased from $35.1 \%$ in 2000 to $45.0 \%$ in 2010 ( $P=.04$ for trend).

\section{4 | Outcomes}

The overall mortality of IE during the study period was $2.8 \%$. Mortality was higher in those with staphylococcal IE in comparison to nonstaphylococcal IE (4.7\% vs. $1.9 \%$; $P=.04$ ) (Table 3). The median length

TABLE 2 Causative organisms of infective endocarditis in US children with and without underlying cardiac condition (2000-2010)

\begin{tabular}{|lll|}
\hline Organism & $\begin{array}{l}\text { Underlying } \\
\text { cardiac } \\
\text { conditions }\end{array}$ & $\begin{array}{l}\text { No underlying } \\
\text { cardiac } \\
\text { conditions }\end{array}$ \\
\hline S. aureus & $28.1 \%$ & $46.9 \%$ \\
\hline Other Staph spp & $6.6 \%$ & $6.3 \%$ \\
\hline Viridans Strep. & $32.7 \%$ & $17.9 \%$ \\
\hline Other Strep & $16.5 \%$ & $9.9 \%$ \\
\hline Gram-negative bacilli & $5.3 \%$ & $7.5 \%$ \\
\hline Polymicrobial & $10.9 \%$ & $11.5 \%$ \\
\hline
\end{tabular}


TABLE 3 Difference in outcomes of US children with Staphylococcal vs. Non-Staphylococcal infective endocarditis ${ }^{a}$ (2000-2010)

\begin{tabular}{|llll} 
Outcomes & $\begin{array}{l}\text { Staphylococcal } \\
\text { IE }\end{array}$ & $\begin{array}{l}\text { Non-Staphylococcal } \\
\text { IE }\end{array}$ & $\begin{array}{l}\boldsymbol{P} \\
\text { value }\end{array}$ \\
\hline LOS & 12 days & 9 days & $<.001$ \\
\hline Complications & & & \\
\hline Acute kidney injury & $10.8 \%$ & $5.5 \%$ & .006 \\
\hline $\begin{array}{l}\text { Stroke } \\
\begin{array}{l}\text { Mechanical } \\
\text { ventilation }\end{array}\end{array}$ & $7.4 \%$ & $4.8 \%$ & .15 \\
\hline \begin{tabular}{l} 
Mortality \\
\hline
\end{tabular} & $4.7 \%$ & $6.8 \%$ & .07 \\
\hline
\end{tabular}

ancludes culture-negative IE cases.

of hospital stay was 10 days (IQR $5-19 d$ ). Median length of stay was longer by 3 days in patients with Staphylococcus species infection compared to those with other causative organisms (12d vs. 9d; $P<.001)$. Median hospital length of stay was not different between those with or without underlying cardiac conditions.

Overall $7.1 \%$ of IE patients had evidence of acute kidney injury (AKI). Proportion of AKI was higher in those with staphylococcal IE vs. non-staphylococcal IE (10.8\% vs. $5.5 \% ; P=.006)$. AKI occurred less often in children with underlying cardiac condition than those without (5.0\% vs. 9.5\%; $P=.02$ ). Mechanical ventilation was used in $7.8 \%$ of all patients. There was a trend for increased use of mechanical ventilation in those with staphylococcal IE compared to others (10.3\% vs. $6.8 \% ; P=.07$ ). Stroke was seen in $5.6 \%$ of patients. The proportion was similar between those with and without cardiac conditions as well as those with staphylococcal IE vs. others. Cardiac valve replacement surgery was performed in $9.2 \%$ of patients. Complications of acute myocardial infarction, CNS abscess, meningitis, encephalopathy, convulsions and ECMO use were too few for meaningful interpretation.

\section{4 | DISCUSSION}

Using nationally representative data our study showed that the mean incidence of infective endocarditis in children was 0.43 per 100000 children with a stable annual incidence during the 11-year study period. Staphylococcus species were the most commonly recovered pathogens from patients with culture-positive IE. Staphylococcal IE was also associated with longer LOS and had the highest mortality rate. However, the proportion of staphylococcal IE showed a decline during the study period.

Infective endocarditis incidence of 0.43 per 100000 children noted in our study is similar to previously reported regional and national estimates that varied between 0.34 to 0.64 per 100000 children. ${ }^{14,15}$ The incidence of IE in the pediatric population is lower than that in adults, which was most recently reported to be 15 per 100000 population. $^{8}$
During the study period there was a stable trend in the incidence of IE in children, which is similar to what Pasquali et al. reported recently. ${ }^{10}$ This is, however, in contrast to the increasing trends of IE in the adult population recently reported by Pant et al. ${ }^{8}$ This increasing incidence has been attributed to an increase in the size of at risk population, such as older, diabetic, and hemodialysisdependent patients. ${ }^{16,17}$

Day et al. had previously described a bi-modal age distribution of IE cases with peaks in infancy and later teens during the period 2000$2003 .^{18}$ In our study, however, the majority of IE cases were in children 11 years and older (56.2\%). There was also a high proportion of IE cases in children without underlying cardiac conditions (46\%) which is similar to previously reported estimates from multicenter hospitalization data in which $58 \%$ of IE cases did not have underlying cardiac condition identified. ${ }^{18}$ This could possibly represent undiagnosed or missed cardiac conditions in children with IE during late childhood. In contrast, Rosenthal et al. reported a decreasing proportion of IE in children with no underlying heart disease from 31\% to $18 \%$ during 1977 to $2004 .^{19}$ However, their data were from a single children's hospital that served as a referral center for children with congenital heart disease leading to sampling bias.

In our study $30.2 \%$ of the hospitalizations were not coded for an organism, which is within the range of previously reported incidence range of 2.5 to $31 \%$ of culture-negative IE. ${ }^{20}$ Most common causes for culture-negative IE are either antimicrobial therapy prior to obtaining blood cultures or IE caused by pathogens that are slow growing or difficult to recover by standard blood culture techniques. We saw a trend towards decline in the incidence of culture-negative IE cases from $35.5 \%$ in 2000 to $18.3 \%$ in 2010 ( $P=.06$ for trend). We speculate that this is likely due to improvement in molecular diagnostic techniques to identify 16s Ribosomal RNA or DNA from tissue or blood. ${ }^{21}$

In a recent study by Day et al. Staphylococci or Streptococci were the predominant IE pathogens identified in more than $90 \%$ of their culture-positive cases. ${ }^{18}$ The majority of our study culture-positive IE cases were caused by Staphylococcus species (43.1\% hospitalizations) followed by Streptococcus species (39.5\%). However, there was a decline in staphylococcal IE over the study period. This is in contrast to recent trend noted in adult IE cases as reported by Pant et al. where they were relative increase in staphylococcal IE of $18.9 \%$ during the period of 2000 to $2011 .^{8}$ We found an increasing trend for streptococcal IE during the study period ( $P$ for trend $=.04$ ). We wanted to assess the impact of the 2007 ACC/AHA IE antibiotic prophylaxis guidelines on rates of streptococcal IE. ${ }^{22}$ The 2007 guidelines limited the antibiotic prophylaxis to specific at risk population as well as prophylaxis prior to gastrointestinal or genitourinary procedures was no longer recommended. We found that the incidence of IE in children during the periods before and after the release of the 2007 guidelines remained unchanged thus providing support in favor of the new guidelines. This is concordant with previously reported studies where no change was noted during similar period $(7,10,23,24)$. However, Pant et al. ${ }^{8}$, using the NIS database found that the incidence of streptococcal IE has 
increased since the release of the new guidelines. Differences in characteristics of patients (adults vs. children) and less need for invasive procedures that could result in bacteremia may account for the differences in results. Further studies are needed to better understand the impact of the guidelines in different populations. This result is concordant with previously reported studies where no change was noted during similar period. ${ }^{7,10,23,24}$

The overall mortality for IE cases in our present study was $2.8 \%$, as compared to previously reported mortality rates of $1.1 \%-18 \%{ }^{10,25}$ Staphylococcal IE was associated with increased mortality that is anecdotally reported in multiple reports, especially with $S$. aureus. ${ }^{4,18,25,26}$ Staphylococcal IE was also associated with significant morbidity with increased use of mechanical ventilation and acute kidney injury.

Although we have used a nationally representative database, our study has important limitations. First, we used ICD-9-CM codes for identification of patients with infective endocarditis. Although, this methodology has been used in both adult and pediatric studies, we cannot exclude variations in coding practices that may have led to over or underestimation of IE incidence rates. Also we were unable to ascertain if these cases fulfilled the Modified Duke criteria for diagnosis of IE as well as further classification of IE. Limited microbiologic data were available with the ICD-9-CM coding and data describing which heart valves were affected could not be ascertained. Possible coding errors as well as lack of information after discharge including total treatment duration pose considerable limitation to the study. Second, as NIS does not have unique patient identifiers, we were unable to identify readmissions or transfers between hospitals. Lastly, the outcome data could not be further subcategorized according to different IE pathogens because of very few numbers of such patients.

\section{CONCLUSIONS}

Our study has several important findings. First, the incidence of IE in children has remained stable during the study period. Second, S. aureus was the commonest causative organism in pediatric IE cases; however, there was a declining trend during the study period. Staphylococcal IE was associated with higher mortality, LOS, AKI, and need for mechanical ventilation. Third, the trend for streptococcal IE related hospitalizations increasing over the study period.

\section{ACKNOWLEDGMENT}

None

\section{AUTHOR CONTRIBUTIONS}

Shipra Gupta has made substantial contributions to conception and design of the study. All authors have made substantial contributions to analysis and interpretation of data. Shipra Gupta has drafted the initial manuscript. All authors have revised the manuscript for important intellectual content and have provided the final approval for the version to be published.

\section{REFERENCES}

[1] Gilboa SM, Salemi JL, Nembhard WN, Fixler DE, Correa A. Mortality resulting from congenital heart disease among children and adults in the United States, 1999 to 2006. Circulation 2010; 122(22):22542263.

[2] Awadallah SM, Kavey RE, Byrum CJ, Smith FC, Kveselis DA, Blackman MS. The changing pattern of infective endocarditis in childhood. Am J Cardiol. 1991;168(1):90-94.

[3] Morris CD, Reller MD, Menashe VD. Thirty-year incidence of infective endocarditis after surgery for congenital heart defect. Jama 1998;279(8):599-603.

[4] Saiman L, Prince A, Gersony WM. Pediatric infective endocarditis in the modern era. J Pediatr. 1993;122(6):847-853.

[5] Weber R, Berger C, Balmer C, et al. Interventions using foreign material to treat congenital heart disease in children increase the risk for infective endocarditis. Pediatr Infect Dis J J. 2008;27(6):544550. Jun

[6] Roig IL, Darouiche RO, Musher DM, Trautner BW. Device-related infective endocarditis, with special consideration of implanted intravascular and cardiac devices in a predominantly male population. Scan J Infect Dis. 2012;44(10):753-760.

[7] Bor DH, Woolhandler S, Nardin R, Brusch J, Himmelstein DU. Infective endocarditis in the U.S., 1998-2009: a nationwide study. PloS One. 2013;8(3):e60033.

[8] Pant S, Patel NJ, Deshmukh A, et al. Trends in infective endocarditis incidence, microbiology, and valve replacement in the United States from 2000 to 2011. J Am Coll Cardiol 2015;1965(19):2070-2076.

[9] Van Hare GF, Ben-Shachar G, Liebman J, Boxerbaum B, Riemenschneider TA. Infective endocarditis in infants and children during the past 10 years: a decade of change. Am Heart J J. 1984;107(6): 1235-1240.

[10] Pasquali SK, He X, Mohamad Z, et al. Trends in endocarditis hospitalizations at US children's hospitals: impact of the 2007 American Heart Association Antibiotic Prophylaxis Guidelines. Am Heart J J. 2012;163(5):894-899.

[11] Baltimore RS, Gewitz M, Baddour LM, et al. Infective endocarditis in childhood: 2015 update: a scientific statement from the American heart association. Circulation 2015;132(15):1487-1515.

[12] Rushani D, Kaufman JS, lonescu-Ittu R, et al. Infective endocarditis in children with congenital heart disease: cumulative incidence and predictors. Circulation 2013;128(13):1412-1419.

[13] Agency for Healthcare Research and Quality. Healthcare Cost and Utilization Project: Introduction to the Nationwide Inpatient Sample (NIS). http://www.hcup-us.ahrq.gov/db/nation/nis/NIS_2009_ INTRODUCTION.pdf. 2009, accessed November 2, 2016.

[14] Coward K, Tucker N, Darville T. Infective endocarditis in Arkansan children from 1990 through 2002. Pediatr Infect Dis J. 2003;22(12): 1048-1052.

[15] Durack DP. RG., editor. Changes in the epidemiology of endocarditis. In: Infective Endocarditis; An American Heart Association Symposium; 1977; Dallas: American Heart Association; pp 3-23.

[16] Boyle JP, Honeycutt AA, Narayan KM, et al. Projection of diabetes burden through 2050: impact of changing demography and disease prevalence in the U.S. Diabetes Care. 2001;24(11):1936-1940.

[17] Wiener JM, Tilly J. Population ageing in the United States of America: implications for public programmes. Int J Epidemiol. 2002;31(4): 776-781.

[18] Day MD, Gauvreau K, Shulman S, Newburger JW. Characteristics of children hospitalized with infective endocarditis. Circulation 2009;119(6):865-870. 
[19] Rosenthal LB, Feja KN, Levasseur SM, Alba LR, Gersony W, Saiman L. The changing epidemiology of pediatric endocarditis at a children's hospital over seven decades. Pediatr Cardiol. 2010;31(6):813-820.

[20] Tunkel AR, Kaye D. Endocarditis with negative blood cultures. N Engl J Med. 1992;326(18):1215-1217.

[21] Bosshard PP, Kronenberg A, Zbinden R, Ruef C, Bottger EC, Altwegg $M$. Etiologic diagnosis of infective endocarditis by broadrange polymerase chain reaction: a 3-year experience. Clin Infect Dis. 2003;37(2):167-172.

[22] Wilson W, Taubert KA, Gewitz M, et al. Prevention of infective endocarditis: guidelines from the American Heart Association: a guideline from the American Heart Association Rheumatic Fever, Endocarditis and Kawasaki Disease Committee, Council on Cardiovascular Disease in the Young, and the Council on Clinical Cardiology, Council on Cardiovascular Surgery and Anesthesia, and the Quality of Care and Outcomes Research Interdisciplinary Working Group. J Am Dental Assoc. 2007;138(6):739-745, 47-60.

[23] Desimone DC, Tleyjeh IM, Correa de Sa DD, et al. Incidence of infective endocarditis caused by viridans group streptococci before and after publication of the 2007 American Heart Association's endocarditis prevention guidelines. Circulation. 2012;126(1):60-64.

[24] Pharis CS, Conway J, Warren AE, Bullock A, Mackie AS. The impact of 2007 infective endocarditis prophylaxis guidelines on the practice of congenital heart disease specialists. Am Heart J. 2011;161(1): 123-129.
[25] Martin JM, Neches WH, Wald ER. Infective endocarditis: 35 years of experience at a children's hospital. Clin Infect Dis. 1997;24(4):669-675.

[26] Johnson JA, Boyce TG, Cetta F, Steckelberg JM, Johnson JN. Infective endocarditis in the pediatric patient: a 60-year singleinstitution review. Mayo Clin Proc Proc. 2012;87(7):629-635.

\section{SUPPORTING INFORMATION}

Additional Supporting Information may be found in the online version of this article.

TABLE S1 ICD-9-CM codes used to identify causative organisms of infective endocarditis in US children (2000-2010)

TABLE S2 ICD-9-CM codes used to identify underlying cardiac conditions in US children with infective endocarditis (2000-2010)

TABLE S3 ICD-9-CM codes used to identify complications of infective endocarditis in US children (2000-2010)

How to cite this article: Gupta S, Sakhuja A, McGrath E, Asmar B. Trends, microbiology, and outcomes of infective endocarditis in children during 2000-2010 in the United States. Congenital Heart Disease. 2017;12:196-201. https://doi.org/10.1111/chd.12425 